\title{
Ação de diferentes maturadores na produtividade e qualidade tecnológica da cana-de-açúcar
}

\author{
Different chemical ripeners action on sugarcane yield and technological quality
}

\author{
Dana Kátia Meschede ${ }^{1}$; Caio Antonio Carbonari ${ }^{2}$, Edivaldo Domingues Velini ${ }^{3}$
}

\begin{abstract}
Resumo - Este trabalho teve por objetivo determinar o efeito de diferentes maturadores, ethephon, trinexapac-ethyl, sulfumeturon-methyl, fluazifop-p-butil e glyphosate, sobre a qualidade tecnológica e produtividade da cana-de-açúcar. O experimento foi conduzido em cana-soca, variedade RB86 - 7515 na Fazenda Santa Antonina, localizada no município de Sertãozinho, estado de São Paulo, no ano de 2007. O delineamento experimental utilizado foi o de blocos completos casualizados com quatro repetições. Os tratamentos consistiram da aplicação dos seguintes produtos: glyphosate $0,40 \mathrm{~L} \mathrm{ha}^{-1}$, sulfumeturon-methyl $20 \mathrm{~g} \mathrm{ha}^{-1}$, sulfumeturon-methyl $10 \mathrm{~g} \mathrm{ha}^{-1}+$ glyphosate $0,20 \mathrm{~L} \mathrm{ha}^{-1}$, fluazifop-p-butil $75 \mathrm{~mL} \mathrm{ha}{ }^{-1}$, trinexapac-ethyl $0,80 \mathrm{~L} \mathrm{ha}^{-1}$, ethephon $0,67 \mathrm{~L} \mathrm{ha}^{-1}$ e testemunha (maturação natural). As avaliações foram realizadas no ponto zero, 15, 30, 45 e 60 dias após aplicação dos produtos (DAA). A aplicação de todos os produtos utilizados como maturadores promoveu efeito significativo, em relação à testemunha, para a característica Pol da cana, desde a primeira época de avaliação (15 DAA) até o final (60 DAA). A altura da planta e o ATR foram alterados pela aplicação dos maturadores dentro dos períodos avaliados. A aplicação de fluazifop-p-butil paralisou o crescimento da cana. A produtividade foi alterada pela aplicação dos maturadores em todas as épocas avaliadas. A aplicação de fluazifop-pbutil aumentou a produtividade, logo após a aplicação (até 15 DAA), no entanto para os demais períodos este tratamento revelou as menores produtividades. Sulfumeturon-methyl, sulfumeturonmethyl + glyphosate, e ethephon apresentaram os melhores resultados de produtividade a partir dos 30 e 60 DAA. Os valores de ATR foram maiores com aplicação de glyphosate e fluazifop-p-butil.
\end{abstract}

Palavras-chave: Saccharum sp, maturação, atributos tecnológicos.

Abstract - This study objectified to determine the effect of the ripeners, ethephon, trinexapac ethyl, sulfumeturon metal, fluazifop-p-butil and glyphosate, over technological quality and sugarcane yield. The experiment was carried out in ratoon cane, (RB86 - 7515 genotype) at Santa Antonina Farm, in Sertãozinho County, São Paulo State, in 2007 agricultural year. The experimental design used was randomized complete block with four replications. Treatments tested were constituted by: glyphosate at $0.40 \mathrm{~L} \mathrm{ha}^{-1}$, sulfumeturon-methyl $20 \mathrm{~g} \mathrm{ha}^{-1}$, sulfumeturonmethyl $10 \mathrm{~g} \mathrm{ha}^{-1}$ glyphosate $+0.20 \mathrm{~L} \mathrm{ha}^{-1}$, fluazifop-p-butyl $75 \mathrm{~mL} \mathrm{ha}^{-1}$, trinexapac-ethyl $0.80 \mathrm{~L} \mathrm{ha}^{-}$ , ethephon $0.67 \mathrm{~L} \mathrm{ha}^{-1}$ and a check without ripeners application. Evaluations were made in the moment of application, 15, 30, 45 and 60 days after products application (DAA). Application of all used products as ripeners promoted significant effect, in relation to the check, for sugar cane Pol characteristic, since the first period of evaluation (15 DAA) until the final (60 DAA). Plant height and ATR were modified by ripeners application inside evaluated periods. Fluazifop-p-butyl

\footnotetext{
${ }^{1}$ Prof. Dra. Faculdades Integradas de Ourinhos - FIO/ Ourinhos -SP

${ }^{2}$ Doutorando em Proteção de Plantas da FCA/UNESP, Botucatu. Faz. Exp. Lageado s/n, Botucatu-SP.

${ }^{3}$ Professor Dr. Da Faculdade de Ciências Agronômicas/UNESP - Botucatu . Faz. Exp. Lageado s/n, Botucatu-SP.
}

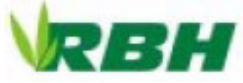


application stopped sugarcane growth. Yield was changed by ripeners applications in all evaluated periods. Fluazifop-p-butyl application increased yield immediately after application (15 DAA), however for the other periods, this treatment showed the lowest yield. Sulfumeturon-methyl, sulfumeturon-methyl + glyphosate and ethephon showed the best yield results from 30 and 60 DAA. ATR values were higher with glyphosate and fluazifop-p-butyl application.

Key-words : Saccharum spp.; ripener; technological attributes

\section{Introdução}

A cana-de-açúcar está entre as principais culturas cultivadas no país, tendo grande importância econômica, sua produção agrícola no ano 2006/2007 foi de aproximadamente 475 milhões de toneladas, das quais 409 milhões na região Centro-Sul do país, sendo São Paulo o principal estado produtor, com $59,9 \%$ da produção nacional (Conab, 2007).

O rendimento econômico da cana-deaçúcar é dado pela produção de sacarose (o componente mais valioso), além de fibra e palha, que pode ser utilizada como fonte de energia para a própria usina.

A produtividade média de cana-deaçúcar, incluindo folhas secas e ponteiros, tem oscilado em torno de 90 toneladas de matéria natural por hectare, mas adotando-se manejo adequado de variedades, de calagem e de adubação, podem-se alcançar produtividades superiores a 150 toneladas de matéria natural por hectare (Oliveira et al., 2001).

Atualmente encontramos variedades melhoradas, com baixos valores de fibras e altos teores de sacarose, o que proporciona maiores produtividades agrícolas e industriais. No entanto, ainda enfrentamos problema de precocidade e florescimento da cultura, não conseguindo atender as demandas com os mesmos teores de Pol exigida pelas indústrias.

Diversos países produtores calculam o rendimento da cana-de-açúcar, através do peso, dos colmos por área de terreno, sendo a produtividade mundial de 73 ton ha ${ }^{-1}$, tendendo a elevar-se com o emprego de novas tecnologias. Outros países estabelecem como rendimento econômico da cultura, a quantidade de açúcar obtida por hectare, contendo os colmos de 7 a $13 \%$ de sacarose, além de 11 a $16 \%$ de fibra.

Segundo Taupier (1999), um hectare de cana-de-açúcar é capaz de produzir 100 toneladas de matéria verde a cada ano, superando duas vezes o rendimento agrícola de outras culturas energéticas; contribui com o equivalente a 10 toneladas de petróleo. O caldo extraído (caldo mesclado) tem por volta de $15^{\circ}$ Brix com uma pureza que flutua entre 80 e $87 \%$. Portanto, 100 t de caldo devem conter 15 $\mathrm{t}$ de sólidos totais e entre 12 e $13 \%$ de Pol. A quantidade de não-açúcares (impurezas) pode variar de 3 a $3 \%$.

Os maturadores, definidos como reguladores vegetais, agem alterando a morfologia e a fisiologia da planta, podendo levar a modificações qualitativas e quantitativas na produção. A utilização dos reguladores vegetais poderá ser uma alternativa compensadora aos investimentos e objetivos propostos à cultura da cana, por possibilitarem incrementos no teor de sacarose, precocidade de maturação e aumento na produtividade (Martins \& Castro, 1999).

Segundo Castro (2000), os principais maturadores químicos utilizados na cana-deaçúcar para restringir seu florescimento são pertencentes ao grupo dos retardadores de crescimento (ethephon, sulfometuron-methyl, trinexapac-ethyl) e do grupo dos inibidores de crescimento (diquat, glyphosate e fluazifop- 
butil). Esses inibidores podem promover uma grande alocação de sacarose para o colmo em curto período de tempo (30 dias), devendo, porém, ser cuidadosamente aplicados para se evitar sobreposição e danos ao canavial, assim como se deve colher a cana tratada com presteza, evitando-se problemas de inversão da sacarose no campo.

A utilização de maturadores e/ou inibidores de florescimento na cultura da canade-açúcar tem como objetivo aumentar a produtividade e antecipar o corte, permitindo, pois, o indispensável manejo da cultura em seu moderno sistema de produção (Pontin, 1995).

Sendo assim, o presente trabalho teve por objetivo avaliar o efeito de maturadores no rendimento e qualidade industrial da cana-deaçúcar.

\section{Material e métodos}

$\mathrm{O}$ experimento foi conduzido em cana soca, variedade RB86-7515, em seu $3^{\circ}$ corte na Fazenda Santa Antonina, localizada no município de Sertãozinho, Estado de São Paulo, pertencente ao Grupo Junqueira Unidade Ribeirão Preto/SP.

$\mathrm{O}$ experimento foi instalado em março de 2007, em solo de textura argilosa. O delineamento experimental utilizado foi o de blocos casualizados com quatro repetições. Cada parcela foi constituída de $150 \mathrm{~m}^{2}$ (5 linhas de $20 \mathrm{~m} \times 1,50 \mathrm{~m}$ ), com área útil de 90 $\mathrm{m}^{2}$ (3 linhas de $20 \mathrm{~m} \times 1,50 \mathrm{~m}$ ). Os tratamentos constituíram-se da aplicação de cinco tipo de maturadores, e uma mistura, e uma testemunha sem aplicação (maturação natural), totalizando 7 tratamentos. Os inibidores de crescimento vegetal foram glyphosate $0,40 \mathrm{~L} \mathrm{ha}^{-1}$, sulfumeturon-methyl $20 \mathrm{~g} \mathrm{ha}^{-1}$, sulfumeturonmethyl $10 \mathrm{~g} \mathrm{ha}^{-1}+$ glyphosate $0,20 \mathrm{~L} \mathrm{ha}^{-1}$, fluazifop-p-butil $75 \mathrm{~mL}$ ha $^{-1}$, trinexapac-ethyl $0,80 \mathrm{~L} \mathrm{ha}^{-1}$, ethephon $0,67 \mathrm{~L} \mathrm{ha}^{-1}$ que são encontrado comercialmente como Roundup original, Curavial, Moddus, Ethrel.

A aplicação dos maturadores foi realizada em 16/04/2007 por meio de pulverizador costal pressurizado $\left(\mathrm{CO}_{2}\right)$ com barra aplicadora, em forma de $\mathrm{T}$, contendo três pontas de pulverização AXI 11002, na pressão de trabalho de 50 PSI e a vazão de $100 \mathrm{~L} \mathrm{ha}^{-1}$.

As avaliações foram realizadas aos 0 , 15, 30 e 60 dias após aplicação (DAA) dos maturadores. Para produtividade, obtida em pré-colheita, uma fileira de plantas foi destinada à mensuração tecnológica, sendo estabelecido $1 \mathrm{~m}$ aleatório a cada época de amostragem, e os colmos coletados encaminhados para o laboratório da usina para serem processados, segundo metodologia do Sistema de Pagamento de Cana pelo Teor de Sacarose, descritas em Fernandes (2003), onde foram considerados os parâmetros: açúcar total recuperável (ATR), sólidos solúveis (Brix em $\%$ ), sacarose (Pol do caldo em \%), fibra industrial na cana (\%) e produtividade $\left(\mathrm{t} \mathrm{ha}^{-1}\right)$.

Os resultados obtidos foram submetidos à análise de variância pelo teste $\mathrm{F}$ e as médias foram comparadas pelo teste t, a nível de $5 \%$ de probabilidade, utilizando o software estatístico SISVAR ${ }^{\circledR}$.

\section{Resultados e discussão}

Na Tabela 1 são apresentados os dados de produtividade e Pol da cana-de-açúcar aos 0 , 15,30 e 60 DAA dos maturadores. Todos os tratamentos testados, exceto a testemunha, apresentaram incrementos significativos na produção, caracterizados em média por $10 \mathrm{tha}^{-}$ 1.

Aos 15 dias após aplicação dos maturadores, revelou-se que o glyphosate e trinexapac-ethyl promoveram queda na produção em relação testemunha. Segundo Rodrigues (1995), após a aplicação de 
glyphosate, a colheita só deve ser efetuada 30 a 60 DAA do maturador, devendo a cana, no momento da aplicação, já estar com seu rendimento agrícola assegurado, pois o produto provocará a paralisação do seu desenvolvimento e conseqüente à redução de produtividade antes do período recomendado. Ainda é possível observar que, o fluazifop-p- butil aos 30 DAA, promoveu redução de produtividade da cana-de-açúcar quando comparado aos demais tratamentos, e este resultado é contrário o que aconteceu na avaliação aos 15 DAA. Através de observação visual, verificou-se que o fluazifop-p-butil provocou necrose dos tecidos da folha do ponteiro da cana-de-açúcar.

Tabela 1. Produtividade e Pol da cana de açúcar avaliada aos 15, 30, e 60 dias após aplicação (DAA) dos maturadores (pré-colheita). Variedade RB86-7515, Fazenda Santa Antonina - Sertãozinho/SP - 2007.

\begin{tabular}{ccccccccc}
\hline \multirow{2}{*}{ Tratamentos } & $\begin{array}{l}\text { Produt. } \\
\text { (ton/ha) }\end{array}$ & Pol (\%) & $\begin{array}{l}\text { Produt. } \\
\text { (ton/ha) }\end{array}$ & Pol (\%) & $\begin{array}{l}\text { Produt. } \\
\text { (ton/ha) }\end{array}$ & Pol (\%) & $\begin{array}{c}\text { Produt. } \\
\text { (ton/ha) }\end{array}$ & Pol (\%) \\
\cline { 2 - 9 } & \multicolumn{2}{c}{ Ponto Zero } & \multicolumn{2}{c}{$15 \mathrm{DAA}$} & $30 \mathrm{DAA}$ & $60 \mathrm{DAA}$ \\
\hline glyphosate & $93,4 \mathrm{a}$ & $8,680 \mathrm{a}$ & $80,02 \mathrm{bc}$ & $10,59 \mathrm{ab}$ & $99,13 \mathrm{ab}$ & $11,71 \mathrm{ab}$ & $99,22 \mathrm{abc}$ & $13,93 \mathrm{a}$ \\
sulf. methyl & $86,0 \mathrm{a}$ & $8,645 \mathrm{a}$ & $96,15 \mathrm{a}$ & $10,68 \mathrm{ab}$ & $99,80 \mathrm{a}$ & $11,64 \mathrm{ab}$ & $104,88 \mathrm{a}$ & $13,34 \mathrm{bc}$ \\
sulf. + glyph. & $93,8 \mathrm{a}$ & $8,675 \mathrm{a}$ & $89,35 \mathrm{abc}$ & $10,23 \mathrm{~b}$ & $103,04 \mathrm{a}$ & $11,40 \mathrm{ab}$ & $103,55 \mathrm{ab}$ & $12,69 \mathrm{c}$ \\
fluazifop & $92,9 \mathrm{a}$ & $8,322 \mathrm{a}$ & $91,75 \mathrm{a}$ & $11,03 \mathrm{a}$ & $88,40 \mathrm{~b}$ & $11,99 \mathrm{a}$ & $95,75 \mathrm{bc}$ & $14,72 \mathrm{a}$ \\
trinexapac & $86,7 \mathrm{a}$ & $8,825 \mathrm{a}$ & $78,85 \mathrm{c}$ & $10,46 \mathrm{~b}$ & $105,04 \mathrm{a}$ & $11,12 \mathrm{~b}$ & $99,37 \mathrm{abc}$ & $13,67 \mathrm{~b}$ \\
etephon & $90,9 \mathrm{a}$ & $8,367 \mathrm{a}$ & $90,77 \mathrm{ab}$ & $10,23 \mathrm{~b}$ & $95,31 \mathrm{ab}$ & $11,21 \mathrm{~b}$ & $99,37 \mathrm{abc}$ & $13,54 \mathrm{bc}$ \\
Testemunha & $94,4 \mathrm{a}$ & $8,285 \mathrm{a}$ & $91,06 \mathrm{ab}$ & $10,17 \mathrm{~b}$ & $99,93 \mathrm{a}$ & $11,70 \mathrm{ab}$ & $93,91 \mathrm{c}$ & $12,68 \mathrm{c}$ \\
\hline F tratamento & $1,22 \mathrm{~ns}$ & 1,55 & $1,81 \mathrm{~ns}$ & $1,91 \mathrm{~ns}$ & $1,53 \mathrm{~ns}$ & $1,14 \mathrm{~ns}$ & $1,60 \mathrm{~ns}$ & $3,71 *$ \\
F Bloco & $1,32 \mathrm{~ns}$ & 0,67 & $1,63 \mathrm{~ns}$ & 5,34 & $0,61 \mathrm{~ns}$ & $0,28 \mathrm{~ns}$ & $2,93 *$ & $2,77 *$ \\
C.V. (\%) & 8,20 & 5,20 & 10,80 & 4,26 & 9,15 & 5,01 & 7,19 & 5,49 \\
D.M.S. & 10,35 & 0,82 & 11,69 & 0,55 & 11,09 & 0,71 & 8,85 & 0,91 \\
\hline
\end{tabular}

Médias seguidas de mesma letra, na coluna, não diferem estatisticamente pelo teste de t, a $5 \%$ de probabilidade.

O teor de sacarose da cana, expresso em Pol do caldo em \%, tive incremento significativo nas plantas que receberam aplicação de maturadores, quando comparados com o ponto zero. Os maiores aumentos observados nos valores de Pol da cana com aplicação de maturadores, em relação á testemunha, foram em para o glyphosate (12\%), sulfumeturon-methyl (7\%), fluazifop-pbutil (20\%), trinexapac-ethyl (10\%) e Ethefon $(8 \%)$. Este resultado, possivelmente deve estar relacionado ao transporte mais rápido da sacarose para o colmo, provocado pela aplicação destes maturadores. Estes resultados corroboram com Romero et al.(2003) e Oliveira et al. (1993), que observaram aumento de sacarose em todas as seções do colmo que receberam aplicação de glyphosate.

Segundo Casagrande (1991), o mecanismo de acúmulo de sacarose é o mesmo, tanto em tecidos imaturos como em adultos, ocorrendo: hidrólise da sacarose, como um pré- 
requisito e limitante da primeira etapa; formação e interconversão de hexosesfosfatos; formação de moléculas análogas à sacarose (talvez, sacarose-P) e acúmulo de parte da sacarose no vacúolo. Todavia, algumas diferenças entre o acúmulo nesses dois tecidos ocorrem como a presença de reguladores vegetais e a ação das invertases.

A altura e o ATR da cana-de-açúcar foram influenciados pela aplicação dos

Tabela 2. Parâmetros de crescimento através da altura da cana de açúcar avaliada ao Zero, 30, e 60 dias após aplicação (DAA) dos maturadores (pré-colheita). Variedade RB86-7515, Fazenda Santa Antonina - Sertãozinho/SP - 2007

\begin{tabular}{|c|c|c|c|c|c|c|c|c|}
\hline \multirow{2}{*}{ Tratamentos } & ATR & Altura & ATR & Altura & ATR & Altura & ATR & Altura \\
\hline & \multicolumn{2}{|c|}{ Ponto Zero } & \multicolumn{2}{|c|}{$15 \mathrm{DAA}$} & \multicolumn{2}{|c|}{$30 \mathrm{DAA}$} & \multicolumn{2}{|c|}{$60 \mathrm{DAA}$} \\
\hline glyphosate & $90,6 \mathrm{a}$ & $2,4 \mathrm{a}$ & $108,01 \mathrm{ab}$ & $2,37 \quad b c$ & $122,73 \mathrm{a}$ & $2,65 a b$ & $138,13 \mathrm{ab}$ & $2,68 \mathrm{bc}$ \\
\hline sulf. methyl & $90,5 \mathrm{a}$ & $2,3 \mathrm{a}$ & $109,13 \mathrm{ab}$ & $2,66 \mathrm{a}$ & $117,41 \mathrm{abc}$ & $2,69 \mathrm{a}$ & $132,71 b c$ & $2,83 \mathrm{a}$ \\
\hline sulf + glypho & $90,7 \mathrm{a}$ & $2,4 \mathrm{a}$ & $105,65 \mathrm{~b}$ & $2,49 a b c$ & $115,31 \mathrm{bc}$ & $2,67 \mathrm{a}$ & $126,89 \mathrm{c}$ & $2,76 \mathrm{ab}$ \\
\hline fluazifop & $87,7 \mathrm{a}$ & $2,4 \mathrm{a}$ & $112,17 \mathrm{a}$ & $2,52 \mathrm{ab}$ & $121,01 \mathrm{a}$ & $2,51 \mathrm{~b}$ & $145,36 \mathrm{a}$ & $2,59 \mathrm{c}$ \\
\hline trinexapac & $91,9 \mathrm{a}$ & $2,4 \mathrm{a}$ & $107,61 \mathrm{ab}$ & $2,30 \quad \mathrm{c}$ & $112,73 \mathrm{c}$ & $2,57 \mathrm{ab}$ & $135,80 \mathrm{~b}$ & $2,64 \mathrm{bc}$ \\
\hline etephon & 87,9 a & $2,3 \mathrm{a}$ & $105,15 \mathrm{~b}$ & $2,51 \mathrm{ab}$ & $113,73 \mathrm{c}$ & $2,61 \mathrm{ab}$ & $134,12 b c$ & $2,72 \mathrm{abc}$ \\
\hline Testemunha & $87,1 \mathrm{a}$ & $2,5 \mathrm{a}$ & $104,65 \mathrm{~b}$ & $2,50 \mathrm{ab}$ & $117,96 a b c$ & $2,67 \mathrm{a}$ & $126,67 \mathrm{c}$ & $2,71 a b c$ \\
\hline F tratamento & $1,53 \mathrm{~ns}$ & 1,55 & $1,92 \mathrm{~ns}$ & $2,09 \mathrm{~ns}$ & $1,56 \mathrm{~ns}$ & $1,36 \mathrm{~ns}$ & $3,73^{*}$ & $1,99 \mathrm{~ns}$ \\
\hline F Bloco & $1,82 \mathrm{~ns}$ & 0,97 & $4,93^{*}$ & $0,59 \mathrm{~ns}$ & $0,93 \mathrm{~ns}$ & $1,91 \mathrm{~ns}$ & $2,60 *$ & $1,76 \mathrm{~ns}$ \\
\hline C.V. (\%) & 3,23 & 5,2 & 3,54 & 6,41 & 5,03 & 4,18 & 5,03 & 4,18 \\
\hline D.M.S. & 4,55 & 0,22 & 4,66 & 0,2 & 7,22 & 0,13 & 8,27 & 0,14 \\
\hline
\end{tabular}

Médias seguidas de mesma letra, na coluna, não diferem estatisticamente pelo teste de t, a $5 \%$ de probabilidade.

Tanto o fluazifop-p-butil como o glyphosate são potentes inibidores do crescimento, o que consequentemente reflete em um menor porte. A inibição ou retardamento do crescimento da cana-deaçúcar, pela aplicação de glyphosate, pode ser devido á inibição do alongamento do caule, que pode também estar relacionada á capacidade da auxina em promover síntese de etileno pelo aumento da atividade da ACCase (Liang et al., 1992). O aumento do etileno pode estimular o maturadores (Tabela 2). Todas as plantas submetidas aos tratamentos continuaram crescendo, desde o ponto zero até a avaliação final, desenvolvida aos 60 DAA. No entanto, as canas que receberam aplicação de fluazifop-pbutil, glyphosate e trinexapac-ethyl, tiveram os menores incrementos de crescimento. A cana submetida a aplicação de fluazifop-p-butil apresentou-se, em média, $15 \mathrm{~cm}$ menor em relação aos demais tratamentos. 
sulfumeturon methyl + glyphosate) foram superiores a testemunha (maturação natural). $\mathrm{O}$ glyphosate e o fluazifop-p-butil promoveram aumentos, em média, de $10 \%$ e $17 \%$,

\section{Conclusões}

Pelos dados analisados é possível inferir que os maturadores influenciaram na qualidade tecnológica da cana-de-açúcar, sendo que o glyphosate e o sulfumeturon ,methyl promoveram as maiores produtividades, assim como os índices de Pol e ATR apresentaram incrementos significativos com aplicação de maturadores.

\section{Referencias}

CASAGRANDE, A.A. Tópicos de morfologia e fisiologia da cana -de-açúcar. Jaboticabal: FUNEP, 1991. 157p.

CONAB, 2007. safra 2007/2008, terceiro levantamento, novembro/2007. / Companhia Nacional de Abastecimento. - Brasília: Conab, 2007. 1. Cana-de-Açúcar. 2. Safra. Acesso: 12/10/2008. Disponível em: www.conab.gov.br/

conabweb/download/safra/3lev-cana.pdf.

CASTRO, P.R.C. Utilização de reguladores vegetais no sistema de produção da cana-deaçúcar. Anais ... Simpósio Internacional de Fisiologia da Cana-de-Açúcar. STAB, Piracicaba, 14 a 20 de outubro de 2000, 10 p. CD-ROM.

FERNANDES, A.C. Cálculos na agroindústria da cana-de-açúcar. Piracicaba: STAB, 2003. 240p.

LIANG, X. et al. The 1-aminociclopropane-1carboxilate synthase genmne family of respectivamente para os valores de ATR, o que refletiu em maiores ganhos econômicos.

Arabidopsis thaliana. Proc. Nalt Acad. Sci., v.89, n.5, p.11046-11050, 1992.

MARTINS, M.B. G, CASTRO, P.R.C. Efeito da giberelina e ethefon na anatomia de plantas da cana de açúcar. Pesq. Agrop. Bras., v.34, n.10, p.1855-1863, 1999.

OLIVEIRA, D.A. et al. Efeito do sulfometuron-methyl em cultura de cana-deaçúcar, cultivada em Podzólico vermelhoamarelo, como maturador vegetal. In: CONGRESSO BRASILEIRO DE HERBICIDAS E PLANTAS DANINHAS 19. 1993, Londrina. Anais... Londrina, 1993 p. 221-223.

OLIVEIRA, M.W et al. Análise quantitativa do crescimento da variedade de cana-de-açúcar RB 2454. In: Encontro de Botânicos de MG, BA e ES. Resumos... XXIII Encontro de Botânicos de MG, BA e ES. UFV/SBB. 1999, v.1, p.89-89.

RODRIGUES, J.D. Fisiologia da cana-deaçúcar. Botucatu: Instituto de Biociências Universidade Estadual Paulista, 1995. 99p. (Apostila).

ROMERO,E.R. et al. Maduración química de los cañaverales: criteios y recomendaciones para implementar un programa de manejo. Av.Agroind., v.24, n.1, p.10-14, 2003.

TAUPIER, L. O. et al. A cana-de-açúcar. In: ICIDCA. Manual dos Derivados da Cana-deAçúcar: diversificação, matérias-primas, derivados do bagaço, derivados do melaço, outros derivados, resíduos, energia. Brasília: ABIPTI, 1999. Cap. 2.1, p.21-27. 